\title{
Anomalous Temperature Dependence of NQR Frequencies
}

\author{
R. J. C. Brown \\ Chemistry Department, Queen's University, Kingston, Ontario K7L 3N6, Canada
}

Z. Naturforsch. 45a, $449-458$ (1990); received August 23, 1989

The anomalous temperature dependence of NQR frequencies which is often observed in crystals in which there is reorientational motion of ions or molecules is considered. Two theories of the phenomena, the jump rotation theory and the pseudo-spin theory, are described, and their successes and failures are discussed. The importance of considering other properties such as pressure dependence of NQR, heat capacity, and elastic constants is emphasized, and it is shown that neither theory is entirely satisfactory at present.

Since the earliest days of nuclear quadrupole resonance, it has been recognized that NQR frequencies depend on temperature, and that in many substances the temperature dependence follows a common pattern: the slope $d v / d T$ is negative, approaches zero at the absolute zero of temperature, and becomes approximately constant at high temperatures. The explanation of this behaviour in terms of librational oscillations of the molecules or ions was proposed by Bayer in 1951 [1]. Several developments of the theory were published shortly thereafter [2-5], and this pattern of temperature dependence became so familiar to workers in the field of NQR that deviation from it was not easily understood. However "anomalous" temperature dependence has been shown to be relatively common, and the subject has been reviewed in a general way several times $[6,7]$.

The objective of this paper is to review certain cases of anomalous temperature dependence in which the anomalies are associated with the rotational motion of ions or parts of molecules or ions, and to try to rationalize the present theoretical models for understanding the NQR and other phenomena. The paper is laid out in three sections. First we discuss what constitutes a normal temperature dependence, and what should be regarded as anomalous. Second, the theory of jump reorientation as it has been applied to NQR is described, together with its successes and limitations. Third, the recently proposed pseudo-spin

\footnotetext{
* Presented at the Xth International Symposium on Nuclear Quadrupole Resonance Spectroscopy, Takayama, Japan, August 22-26, 1989.

Reprint requests to Prof. R. J. C. Brown, Department of Chemistry, Queen's University of Kingston, Kingston, Ontario K7L 3N6.
}

theory of ammonium perrhenate is described, and its extension to other salts is considered. The review of the literature has been carried out with these objectives in mind, and is not exhaustive.

The experimental data have been well summarized in the reviews in references [6] and [7], and in the papers describing the various theories. Studies on several ammonium salts are presently in progress [8]. The most notable anomalies are very large positive temperature coefficients, associated with positive pressure coefficients in at least some cases, and with anomalous thermal expansion and heat capacity.

In assessing any theory which purports to account for a set of observations, it is important not to take too narrow a view. Crystals which show anomalies in their NQR properties usually have other anomalous properties as well, and it is reasonable to suppose that the anomalies are related. It is hardly satisfactory to explain the NQR temperature dependence in terms of some model which either makes no predictions about other interesting properties or, worse, is inconsistent with the observed properties. It is also unsatisfactory to place too much reliance on a theory which is specific to one compound only, and fails to account for the properties of other related compounds. Several of the compounds which show anomalous temperature dependences have been studied in great detail, and it is possible to test various aspects of any theory against a variety of experimental results. Indeed, it is important to do so in order that NQR may maintain its place as one of the useful diagnostic tools of solid state science.

These statements may seem redundant, and yet the theories which are currently used to explain anomalous temperature dependences will be shown to be some- 
what unsatisfactory by these criteria. It is often made to appear that unusual experimental observations are understood in terms of some limited theory, but such a claim may serve only to detract from the interest of truly anomalous observations by making them seem normal.

\section{Anomalous versus Normal Temperature Dependence}

If we are to recognize an anomalous temperature dependence, then it is necessary first to describe what is normal. The simplest description is the "Bayertype" temperature dependence, in which $\mathrm{d} \ln v / \mathrm{d} T$ is approximately $-1 \times 10^{-4} \mathrm{~K}^{-1}$ over most of the temperature range, gradually becoming more negative as the temperature increases (i.e. $\mathrm{d}^{2} \ln v / \mathrm{d} T^{2}$ is small and negative), and approaching zero at the lowest temperatures. Such behaviour was explained by Bayer [1], who showed that torsional, or librational, modes of oscillation of polyatomic molecules or ions in a crystal would lead to an averaging of the principal component of the electric field gradient (EFG) at the nuclear site according to the formula

$$
q=q_{0}\left\{1-\frac{3}{2}\left\langle\vartheta^{2}\right\rangle\right\}
$$

where $q_{0}$ is the EFG in the stationary molecule or ion, and $\left\langle\vartheta^{2}\right\rangle$ is the mean squared angle of displacement. The temperature dependence of $v$, which is proportional to $q$, then reflects the temperature dependence of $\left\langle\vartheta^{2}\right\rangle$, which is determined by the (angular) frequency $\omega_{1}$ of the appropriate librational mode, the moment of inertia $I$ corresponding to that motion, and the temperature. From the statistical mechanics of a harmonic oscillator,

$$
\left\langle\vartheta^{2}\right\rangle=\frac{\hbar}{2 \omega_{1} I} \operatorname{coth}\left(\frac{\hbar \omega_{1}}{2 k T}\right),
$$

These two equations predict that $\mathrm{d} v / \mathrm{d} T$ is negative and constant at high temperature, and approaches zero as the temperature approaches zero. In many cases, it allows calculation of $\mathrm{d} v / \mathrm{d} T$ from spectroscopic and structural data to a reasonable degree of accuracy. But while this is a qualitatively correct picture, the explicit temperature dependence shown is hardly ever obeyed exactly, because both $q_{0}$ and $\omega_{1}$ are affected by thermal expansion and so are functions of temperature. The EFG contains both local and inter-molecular contributions, of which the latter are sensitive to details of the crystal structure; the librational mode frequencies are "external" modes which are determined largely by intermolecular interactions and are sensitive to the unit cell geometry.

The effect of thermal expansion on $\omega_{1}$ is, in many cases, to cause $\omega_{1}$ to decrease as the temperature increases, and a satisfactory account can often be given by assuming a linear dependence of $\omega_{1}$ on temperature, at least over a moderate temperature range [5]:

$$
\omega_{1}=\omega_{1}^{0}\left\{1-g_{1}\left(T-T^{0}\right)\right\} .
$$

There is experimental support for this assumption from Raman spectroscopy, and the method has been used in many cases to explain the small negative values of $\mathrm{d}^{2} v / \mathrm{d} T^{2}$ which are commonly observed. Curvature of this sort is therefore not to be considered anomalous.

The variation of $q_{0}$ with the unit cell dimensions can lead to large contributions to $\mathrm{d} v / \mathrm{d} T$ through thermal expansion. The only way to examine these contributions is through study of the pressure dependence of $v_{q}$. The basic theory and experimental techniques were pioneered by Kushida, Benedek and Bloembergen [4], and there have been many experimental studies made since then which have helped to understand anomalous temperature dependences. The NQR frequency is taken to be a function of a geometrical variable as well as the temperature, and the second variable is most chosen to be the volume. It follows that

$$
\left(\frac{\partial v}{\partial T}\right)_{P}=\left(\frac{\partial v}{\partial T}\right)_{V}-\frac{\alpha}{\beta}\left(\frac{\partial v}{\partial P}\right)_{T},
$$

where $\alpha$ is the bulk thermal expansion and $\beta$ is the isothermally compressibility. The first term on the right hand side is usually identified with the Bayer temperature dependence, on the assumption that if the volume of the crystal is constant, then $q_{0}$ and $\omega_{1}$ will also be constant and the Bayer theory can be relied upon. It may be noted in passing that application of pressure affects $\omega_{1}$ as well as $q_{0}$, and the separation of the two effects is not straightforward.

There are limitations to this approach, however, because the relevant properties of a crystal are usually dependent on the individual unit cell dimensions (and perhaps angles) and on the atomic positions within the unit cell. The situation has been summarized previously [9] and has not changed since then, because application of uniaxial stress has not proven to be a practical technique in NQR. But anisotropy of the various properties of non-cubic ammonium salts turns 
out to be a central feature of their anomalous behaviour, and in a sense NQR must be judged to be limited in its ability to explore these properties fully.

On the other hand, as long as one is content with qualitative trends, then (4) allows us to understand a number of compounds in which $\mathrm{d} v / \mathrm{d} T$ has unexpected, even positive, values. For many crystals, $\alpha / \beta$ has a value laying in the range $1-3 \mathrm{MPa} / \mathrm{K}$, and hence over a group of compounds a linear correlation between $(\partial v / \partial T)_{P}$ and $(\partial v / \partial P)_{T}$ is expected, and found [10]. This allows us to classify many positive temperature dependences as "normal", as long as they can be accounted for within the framework of (4), with a negative pressure coefficient $(\partial v / \partial P)_{T}$. Cases which cannot be fitted to this equation are "anomalous", because they require an extension to, or modification of, the two-variable theory; in the case of $\mathrm{NH}_{4} \mathrm{ReO}_{4}$, the volume of the crystal is almost entirely irrelevant to the NQR problem.

\section{The Jump Reorientation Theory}

In 1979, Negita, N. Nakamura, and Chihara proposed a theory of anomalous temperature dependences in crystalline ammonium salts [11], and since then there have been several further accounts and applications of the theory [7, 12-18]; it has recently been applied outside the field of NQR to provide a model for a phase transition [19].

In this model, it is assumed that the ammonium ion spends part of its time localized within a rotational potential well, perhaps $\mathrm{H}$-bonded to nearest neighbour anions, and for the remainder of the time is in a state of transition as it rotates from one stable orientation to another. The observed effect of the ammonium ion on a neighbouring quadrupolar nucleus is a time average of the contributions of the ammonium ion in these two states of motion to the EFG at the nuclear site. This is a two-state theory: the ammonium ion is either localized or else it is rotating. If the ammonium ion spends an average time $\tau_{r}$ in a single orientation in a potential well, and the transition from one orientation to another takes a time $\tau_{t}$, then the average effect on the NQR frequency is

$$
v=v_{0}(T)+\frac{v_{r} \tau_{r}+v_{t} \tau_{t}}{\tau_{r}+\tau_{t}}
$$

where $v_{0}(T)$ is the NQR frequency in the absence of the cation, including the Bayer temperature depen- dence if appropriate, and $v_{r}$ and $v_{t}$ represent the increments to the NQR frequency due to the cation in the stationary and rotating states, respectively. An equivalent form is

$$
v=v_{0}(T)+v_{r}+\Delta v \frac{\tau_{t}}{\tau_{r}+\tau_{t}},
$$

where $\Delta v=v_{t}-v_{r}$ is the change in the NQR frequency when the ion is in the process of making a rotational jump from one potential well to another. In most of the applications so far, $\Delta v$ has been positive, but there seems no reason why the theory should be restricted to positive-slope anomalies. Indeed, in $\left(\mathrm{NH}_{4}\right)_{2} \mathrm{TeI}_{6}$ in which there are three NQR lines with anomalous temperature dependences, two of the lines are assigned negative values of $\Delta v$; all three lines can then be fitted to the model with a single $\tau_{r}[18]$.

Jump reorientation of polyatomic ions in crystals is not by any means a new idea, at least for temperatures and barrier heights where tunnelling can be neglected. It is supported by neutron scattering studies [20] and by somewhat more indirect evidence derived from spin-rotation relaxation in the NMR of low-barrier salts [21]. What is special about the theory is the assumption of a direct observable influence of the ammonium ion on the NQR frequency in its "transition state" between stable orientations; if the theory is valid, it means that NQR is one of the few spectroscopic ways of probing the properties of the transition state. The theory differs from the hypothesis of "partial scission of $\mathrm{H}$-bonds" due to thermally excited vibrations and thermal expansion described by D. Nakamura et al. [6], in which there is no mention of the effect of reorientation through a transition state.

In fitting the model to NQR, several procedures have been used. One way is to use NMR relaxation times to determine the rotational correlation time, which can be interpreted either as $\tau_{r}[11]$, or as $\left(\tau_{r}+\tau_{t}\right)$ [15]; the time required for the rotational jump, $\tau_{t}$, may be assumed to be fixed [11], or may be permitted to vary with temperature [15]. The model is generally successful in fitting the NQR temperature dependences, at least qualitatively.

The relationship between the jump model and the thermodynamic properties of the ammonium salts concerned has never been discussed in detail. The model is a "two-state" model: the $\mathrm{NH}_{4}^{+}$ion is either at rest or rotating. Yoshioka et al. [15] assumed that the two states were associated with single energy levels so 
that the relative populations of these two states must be related by a Boltzmann factor:

$$
n_{t} / n_{r}=\exp (-\Delta E / R T),
$$

where $\Delta E$ is the energy difference between the two states. The populations of each of the two levels are also proportional to the lifetimes of the ion in the two states:

$$
\frac{n_{t}}{n_{r}}=\frac{\tau_{t}}{\tau_{r}}
$$

These equations suggest that the Boltzmann factor also governs the lifetimes, from which it follows that at high temperatures $\tau_{r}$ cannot be shorter than $\tau_{t}$. For several salts to which the model has been applied, $\tau_{t}$ is approximately $2 \times 10^{-12} \mathrm{~s}$, and $\tau_{r}$ is found to be shorter than this at the high temperature end of the experiments [7].

This suggests that it is a more satisfactory approach to assume that each of the two states has an associated complex of energy levels so that a thermodynamic or quasi-chemical approach is appropriate:

$$
n_{t} / n_{r}=K(T)=\exp \left(-\Delta G^{0} / R T\right),
$$

where $K(T)$ is an equilibrium constant and $\Delta G^{0}$ is a Gibbs energy difference. In the high temperature limit, the ratio of populations (and of lifetimes) is equal to $\exp \left(\Delta S^{0} / R\right)$ and can exceed unity.

This approach can be extended using ideas taken from a recent thermodynamical analysis of ammonium salts by Johnson [22,23] in which the difference between ammonium salts and the corresponding rubidium salts is considered. It was shown that for a wide variety of crystalline salts $\left(\mathrm{NH}_{4}\right)_{n} \mathrm{X}$, the quantity

$\Delta(X)=\frac{1}{n}\left\{\Delta_{\mathrm{f}} H^{0}\left[\left(\mathrm{NH}_{4}\right)_{n} \mathrm{X}\right]-\Delta_{\mathrm{f}} H^{0}\left[\mathrm{Rb}_{n} \mathrm{X}\right]\right\}$

for various anions $\mathrm{X}^{n-}$ satisfies the relation

$$
\Delta(X)+E_{a}(X)=143 \mathrm{~kJ} / \mathrm{mol}
$$

within a few $\mathrm{kJ} \mathrm{mol}^{-1}$, where $E_{a}(X)$ is the barrier to ammonium ion rotation in the corresponding salt. This empirical observation is supported by a detailed Born-Harber cycle analysis and other thermochemical calculations. Johnson is sufficiently sure of his correlation that he suggests that in the case of the one exception to the scheme, namely $\mathrm{NH}_{4} \mathrm{ReO}_{4}$, the NBS thermochemical data are incorrect, and gives estimated corrected values for $\Delta_{\mathrm{f}} \mathrm{H}^{0}$ of $\mathrm{NH}_{4} \mathrm{ReO}_{4}$ and $\mathrm{RbReO}_{4}$.
It is concluded that ammonium salts are stabilized by a contribution to the lattice energy due to the nonspherical cation, and that this stabilization energy is measured by $E_{a}$. Hence, if the ammonium ion were in a state of free rotation, and as a result behaved as a spherical ion, then the crystal enthalpy would be higher by an amount $E_{a}$. In other words, $E_{a}$ can be interpreted as a measure of the enthalpy difference $\Delta H^{0}$ between a hypothetical state of the crystal in which the $\mathrm{NH}_{4}^{+}$ions are rotating freely, and the actual state of the crystal.

The $\Delta S^{0}$ between the freely rotating state and the localized librational state can be estimated from statistical mechanics. In the freely rotating state,

$$
S_{t}=R \ln \left\{8 \pi^{2} e(2 \pi I k T)^{3 / 2} / \sigma h^{3}\right\},
$$

and in a state of librational motion with three degrees of freedom, each degree of freedom contributes:

$$
S_{r}(i)=R\left\{x /\left(e^{x}-1\right)-\ln \left(1-e^{-x}\right)\right\},
$$

where $x=\hbar \omega_{1} / k T$ for each of the three librational frequencies. Inserting the appropriate values for the ammonium ion in $\mathrm{NH}_{4} \mathrm{ReO}_{4}, I=4.74 \times 10^{-47} \mathrm{~kg} \mathrm{~m}^{2}$, $\sigma=12$, and torsional frequencies of $276.2 \mathrm{~cm}^{-1}\left(A_{g}\right)$ and $265.9 \mathrm{~cm}^{-1}\left(E_{g}\right)$ [24], we find at $300 \mathrm{~K}$

$$
\Delta S^{0}=S_{t}-\sum S_{r}(i)=16.7 \mathrm{~J} \mathrm{~K}^{-1} \mathrm{~mol}^{-1} \text {. }
$$

Hence at $300 \mathrm{~K}$, with $\Delta H^{0}=9.0 \mathrm{~kJ} \mathrm{~mol}^{-1}$ [25], we find $\Delta G^{0}=4.0 \mathrm{~kJ} \mathrm{~mol}^{-1}$, and $n_{t} / n_{r}=0.2$. From the figures given by Chihara et al. based upon NQR data, this ratio is 2.7 [16], so the agreement is not good; in order to match this value, a much larger $\Delta S^{0}$ value, $45 \mathrm{~J} \mathrm{~K}^{-1} \mathrm{~mol}^{-1}$, is required in order to bring the condition $K=1$ at temperature $T=\Delta H^{0} / \Delta S^{0}$ close to $200 \mathrm{~K}$ where the inflection in the NQR data is found. Yet the estimate of $\Delta S^{0}$ given in (13) is too high, if anything, since in transition state theory only one degree of freedom is considered to be converted from vibration to unhindered motion.

The thermodynamic model also permits us to calculate the contribution to the heat capacity. For when the above quasi-chemical equilibrium shifts as a result of a change in temperature, there is a contribution to the heat capacity given by

$$
\Delta C_{p}=R\left(\frac{K}{(K+1)^{2}}\right)\left(\frac{\Delta H^{0}}{R T}\right)^{2} .
$$

The second factor has a maximum at the temperature at which $K=1$. Using the value $\Delta S^{0}=16.7 \mathrm{~J} \mathrm{~K}^{-1}$ 
$\cdot \mathrm{mol}^{-1}$, this predicts a maximum heat capacity contribution of $8 \mathrm{~J} \mathrm{~K}^{-1} \mathrm{~mol}^{-1}$ at about $540 \mathrm{~K}$. Alternatively, using $\Delta S^{0}=45 \mathrm{~J} \mathrm{~K}^{-1} \mathrm{~mol}^{-1}$, a maximum $\Delta C_{p}$ of $60 \mathrm{~J} \mathrm{~K}^{-1} \mathrm{~mol}^{-1}$ is found at $200 \mathrm{~K}$, where the maximum is found experimentally. This is somewhat larger than the observed excess $C_{p}$ value [26], but is of the right order of magnitude.

We see that if the jump model is to be used, it makes sense to use a thermodynamic approach, for it allows correlation with at least the heat capacity; inclusion of the thermal expansion and its anisotropy is also a possibility.

The jump model has been criticized in the case of $\mathrm{NH}_{4} \mathrm{ReO}_{4}$, on the grounds that it is inconsistent with the measured pressure dependences of both the NQR frequency and the proton $T_{1}$ [16]. The argument is that common sense and experimental measurement show that the ammonium ion rotational motion slows down under high hydrostatic pressure, and the model therefore predicts a negative value for $(\partial v / \partial P)_{T}$, in contradiction to one of the most striking properties of $\mathrm{NQR}$ in $\mathrm{NH}_{4} \mathrm{ReO}_{4}$. The details of the argument are to be found in [16].

There has been no attempt to fit the temperature dependence of $v$ in $\mathrm{NH}_{4} \mathrm{IO}_{4}$ to the jump model. In this salt, $v$ starts from near zero and rises rapidly, with an inflection near $200 \mathrm{~K}$ [27]; there is no underlying Bayer-type curve. The proton relaxation measurements show that the $\mathrm{NH}_{4}^{+}$ion dynamics are very similar to those in the perrhenate [28], and so a similar value of $\tau_{t}$ should result. The behaviour of $\mathrm{NH}_{4} \mathrm{TcO}_{4}$ is also anomalous [8] and offers an interesting case for testing any theory because the thermal expansion, though anomalous, differes markedly from that in the perrhenate and periodate [29].

Replacement of the ammonium ion by alkyl- and aryl-substituted Group V cations yields perrhenates and periodates with a variety of phase transitions and anomalous temperature dependences, which have been studied by Burkert and co-workers [30-34]. A very large negative $(\partial v / \partial T)_{P}$ is found in $\mathrm{N}\left(\mathrm{CH}_{3}\right)_{4} \mathrm{IO}_{4}$ [30] and in $\mathrm{P}\left(\mathrm{CH}_{3}\right)_{4} \mathrm{ReO}_{4}$ [32] which are as spectacular as the positive slopes in the ammonium salts. These results have not been analysed by any fundamental model and offer the possibility of determining the effect of internal motion as well as of the overall rotation of the cation. In some of these cases, $\eta$ is large and $I>3 / 2$, and there is the possibility of anomalous temperature dependence of the individual transition frequencies through variations in the $x x$ and $y y$ com- ponents of the EFG, as well as through the $z z$ component.

In hexahydrated hexachlorostannates of divalent metals, a group of related anomalous temperature dependences is found. The first report was of the $\mathrm{Ca}$ salt [35], and the $\mathrm{Mg}, \mathrm{Mn}, \mathrm{Co}$, and Ni salts were studied in protonated and deuterated forms later [36]. Study of the ${ }^{35} \mathrm{Cl}$ and ${ }^{1} \mathrm{H}$ relaxation times in the $\mathrm{Ca}$ salt as a function of temperature permitted identification of three distinct modes of motion of the cation, as well as anion reorientation [14]. Analysis of the NQR temperature dependence using the jump model $[14,16]$ identified the $180^{\circ}$ flips of the water moleules as the motion responsible for the anomaly. The other salts show similar temperature dependences although with larger frequency shifts, and probably can be fitted using similar dynamical parameters. It is notable that the deuteration shift changes sign in the middle of the temperature range in all three salts for which data is available.

The salts $\mathrm{NH}_{4} \mathrm{ClO}_{4}$ and $\mathrm{NH}_{4} \mathrm{BF}_{4}$ are both of orthorhombic rather than tetragonal structure and show anomalous temperature dependences $[8,37]$. There has been intense study of the perchlorate by various techniques because of controversy over the existence of phase transitions, and the fluoborate presents a similar problem.

One of the earliest applications of the jump model was to $\left(\mathrm{NH}_{4}\right)_{2} \mathrm{SnCl}_{6}$ [13]. In this and several isostructural salts, $(\partial v / \partial T)_{P}$ is negative, but there is an inflection in the temperature dependence curve at about $120 \mathrm{~K}$, above which $\left(\partial^{2} v / \partial T\right)_{P}$ is positive. The effect is small, but constitutes a real deviation from the normal pattern. A second explanation of the anomaly has been given in terms of a highly anharmonic potential function for the anion librational mode [38]. The properties of $\left(\mathrm{NH}_{4}\right)_{2} \mathrm{PbCl}_{6}$ and $\left(\mathrm{NH}_{4}\right)_{2} \mathrm{TeCl}_{6}$ are very similar except that there is a phase transition $[39,40]$.

The $\mathrm{SnCl}_{6}^{2-}$ librational mode in $\left(\mathrm{NH}_{4}\right)_{2} \mathrm{SnCl}_{6}$ is not observable spectroscopically but can be measured by inelastic neutron scattering [41]. The results show that both of the previous explanations of the NQR anomaly are redundant. It is found that at the Brillouin zone centre the librational frequency decreases as the temperature is lowered, approximately following the equation

$$
\omega_{1}=2 \pi \sqrt{a+b T},
$$

where $a=4.0 \times 10^{23} \mathrm{~Hz}^{2}$ and $b=2.6 \times 10^{21} \mathrm{~Hz}^{2} \mathrm{~K}^{-1}$. The frequency does not reach zero, and there is no 


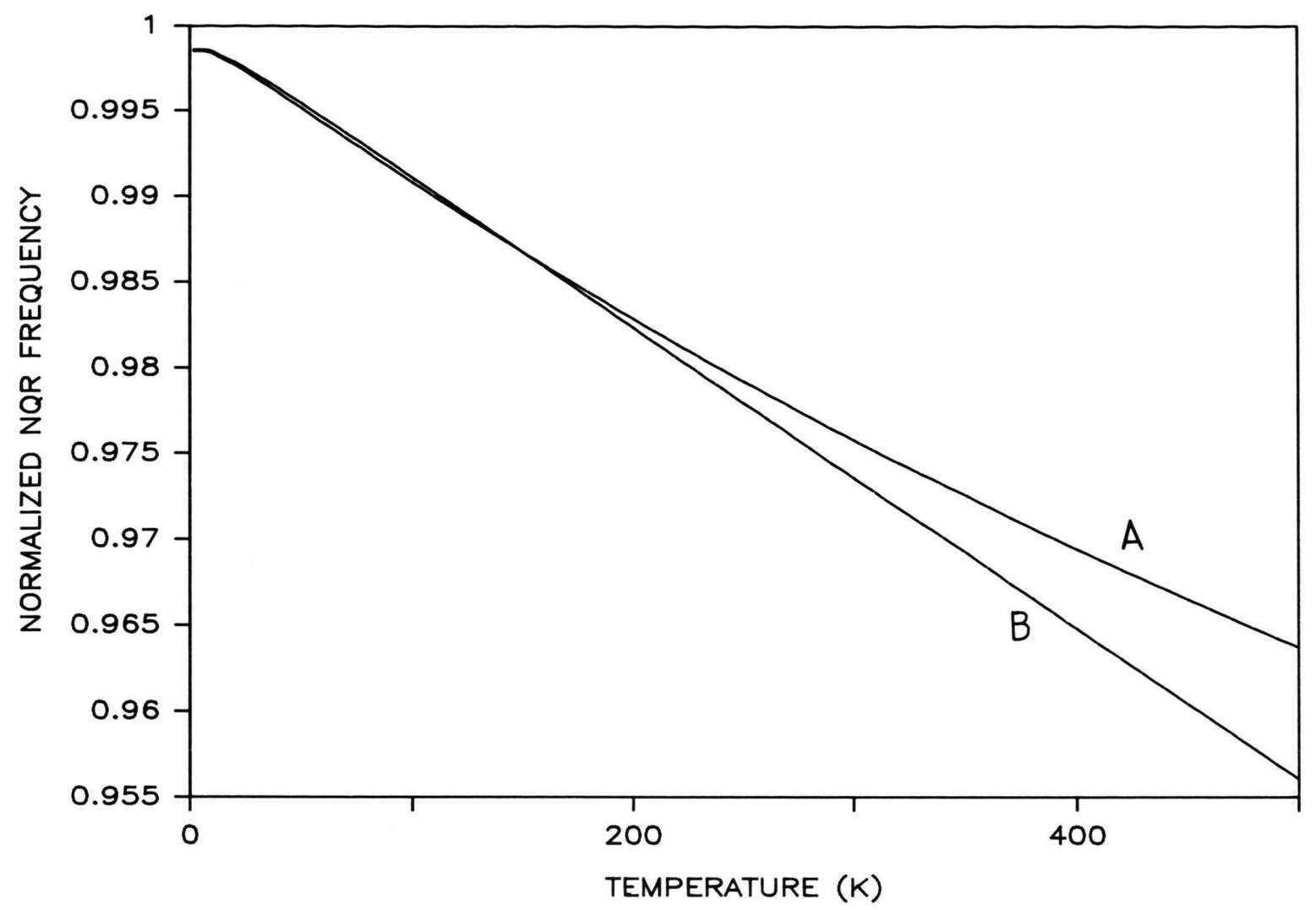

Fig. 1. Curve A: Simulated temperature dependence resulting from a soft librational mode described by (15), (1) and (2) with $a=4.0 \times 10^{23} \mathrm{~Hz}^{2}$ and $b$ set equal to an assumed average over the Brillouin zone, $b_{\text {eff }}=2.6 \times 10^{20} \mathrm{~Hz}^{2} \mathrm{~K}^{-1}$. Curve $\mathrm{B}$ : Temperature dependence of a "non-soft" mode with $b=0$ and $a=4.4 \times 10^{23} \mathrm{~Hz}^{2}$, a value chosen to give (visually) the same average slope below about $150 \mathrm{~K}$.

phase transition. On the other hand, near the zone edge, the frequency is independent of temperaure. The mean square librational amplitude $\left\langle\vartheta^{2}\right\rangle$ reflects an average over the whole zone, and so the effective temperature dependence of the librational frequency is much smaller than is indicated by the above value of $b$; if we assume that $b_{\text {eff }}=b / 10$, and calculate $\left\langle\vartheta^{2}\right\rangle$ as a function of temperature, then the result shown in Fig. 1 is obtained. While this assumption is only an approximation, the curve agrees qualitatively with the NQR temperature dependence. The temperature dependence of the ${ }^{35} \mathrm{Cl} T_{1}$ [13] can presumably be ascribed to the $\omega^{-5}$ dependence found in the anharmonic Raman process [42]. There are features in the ${ }^{35} \mathrm{Cl} T_{1}$ data for $\left(\mathrm{NH}_{4}\right)_{2} \mathrm{PbCl}_{6}$ and $\left(\mathrm{NH}_{4}\right)_{2} \mathrm{TeCl}_{6}$ corresponding to the phase transition $[39,40]$, which supports this idea. This explanation of the properties of these salts, based upon experimental observation of a soft mode, seems preferable to both the jump model and the simple anharmonic model.

The results on pentachloroethylstannate salts reported recently appear to reflect the internal motion of the anion in the NQR temperature dependence [43].

In a curious negative example, the temperature dependence of the NQR frequency in pyridinium tetrachloroaurate(III) is perfectly normal, but the ${ }^{35} \mathrm{Cl} T_{1}$ below $350 \mathrm{~K}$ follows the ${ }^{1} \mathrm{H} T_{1}[44,45]$, indicating a relationship between the quadrupolar relaxation and the reorientation of the cation about its pseudo- $\mathrm{C}_{6}$ axis. The fluctuating part of the EFG responsible for the relaxation is estimated as 0.075 times the static EFG, which corresponds to $2.1 \mathrm{MHz}$. Use of this value for $\Delta v$ in (5') together with estimates of $\tau_{c 1}$ from NMR [45] and $\tau_{t}=2 \times 10^{-12} \mathrm{~s}$ suggests that there should be an anomalous shift of $220 \mathrm{kHz}$ in the NQR frequency at $350 \mathrm{~K}$, which is not observed. On the 
other hand, the pyridinium hexahalometallates do show anomalous temperature dependences [46-48].

To summarize, the jump model is successful in some cases in describing the anomalous temperature dependence, but it suffers from inconsistencies and difficulties if regarded as an accurate physical model of the fundamental process affecting NQR frequencies. It may be a convenient parametrization of the problem, with a plausible model used as a conceptual support, but a more sophisticated approach is required in the long run.

\section{The Pseudo-Spin Model for $\mathrm{NH}_{4} \mathrm{ReO}_{4}$}

Recently a model for the properties of $\mathrm{NH}_{4} \mathrm{ReO}_{4}$ based upon cooperative ordering of the ammonium ions was proposed $[49,50]$. The theory treats the problem by means of a pseudo-spin formalism and assumes that there is a coupling between the orientation of the ammonium ions and the distortion of the unit cell. Using the mean field approximation, a complete solution is obtained, and a good account is given of the NQR temperature dependence, the anisotropic thermal expansion, and the heat capacity; the temperature dependence of one of the elastic constants is also predicted. The agreement between theory and experiment is striking, and further experimental tests are in progress.

The theory depends upon the fact that in the scheelite structure the ammonium ion is situated at a site of $\mathrm{S}_{4}$ symmetry surrounded by two sets of four equidistant oxygen atoms in early tetrahedral coordination [51]; all oxygen sites are crystallographically equivalent, but relative to a given ammonium ion the eight nearby oxygen sites are of two distinct types, and correspondingly there are two $\mathrm{N}-\mathrm{O}$ distances. Each of the eight oxygen atoms adjacent to a given ammonium ion can be classified as "equatorial" and "axial", depending on the position of the centre of the corresponding anion relative to the reference ammonium ion. There are two possible orientations of an ammonium ion, in which the $\mathrm{N}-\mathrm{H}$ bonds point in the direction of either the axial or equatorial oxygen atoms. The axial sites are about $0.1 \AA$ closer to the nitrogen atom than are the equatorial sites (in $\mathrm{NH}_{4} \mathrm{ReO}_{4}$ ), and it is the axial orientation which is found at low temperature in the deuterated salt [51]. In the pseudo-spin theory, it is assumed that there is significant occupation of the (higher energy) equatorial orientation at higher temperatures, and that the energy difference between the two orientations is a function of the unit cell dimensions.

The pseudo-spin theory does not take any account of the kinetic energy of the reorientation ammonium ion and therefore stands in contrast to the theory of thermal expansion discussed by Smith [52], in which the quantized rotational levels are assumed to be functions of the unit cell dimensions. The quantum calculation was not brought to the point of numerical results in the cases of tetragonal and orthorhombic crystals.

Further, in the pseudo-spin theory the NQR frequency is assumed to be a function of the unit cell dimensions alone. Thus the theory is a static theory of the anomalous temperature dependence, whereas the jump model is a dynamic theory. In support of this approach, it may be recalled that it was shown in an early publication [53] that for scheelite-type perrhenates, a linear correlation between $v$ and the $a$-parameter accounts for variations of $v$ with temperature, pressure, and the cation in a qualitative way. On the other hand, it has been shown that $v$ is a very sensitive function of the atomic position coordinates, especially the orientation of the anion in the unit cell [54]; these "internal coordinates" cannot be controlled independently.

A pseudo-spin variable $\sigma^{\mathrm{z}}(i)$ with eigenvalues \pm 1 is used to represent the two orientations of each $\mathrm{NH}_{4}^{+}$ ion. The changes in the tetragonal unit cell dimensions can be represented by the strains $\varepsilon_{i j}$ of elasticity theory. Since there is no change of symmetry, the strains can be represented entirely in terms of the following linear combinations of strains, which are normalized as spherical harmonics:

$$
\begin{aligned}
& \varepsilon_{00}=\varepsilon_{x x}+\varepsilon_{y y}+\varepsilon_{z z}, \\
& \varepsilon_{20}=(\sqrt{3} / 6)\left(2 \varepsilon_{z z}-\varepsilon_{x x}-\varepsilon_{y y}\right) .
\end{aligned}
$$

The first of these measures the volume change, and the second the distortion. In $\mathrm{NH}_{4} \mathrm{ReO}_{4}, \varepsilon_{00}$ hardly changes with temperature, while $\varepsilon_{20}$ changes dramatically, so it is assumed that all the phenomena can be accounted for using only $\varepsilon_{20}$. The energy of an ammonium ion is assumed to be a linear function of $\varepsilon_{20}$ with a coefficient $V_{s}$.

For a crystal with $N$ unit cells, each of volume $\Omega$, the Hamiltonian is written (after some manipulations)

$$
\mathscr{H}=-\sum\left(\Delta_{0}+V_{s} \varepsilon_{20}\right) \sigma^{z}(n)+\frac{1}{2} N \Omega c_{0} \varepsilon_{20}^{2},
$$




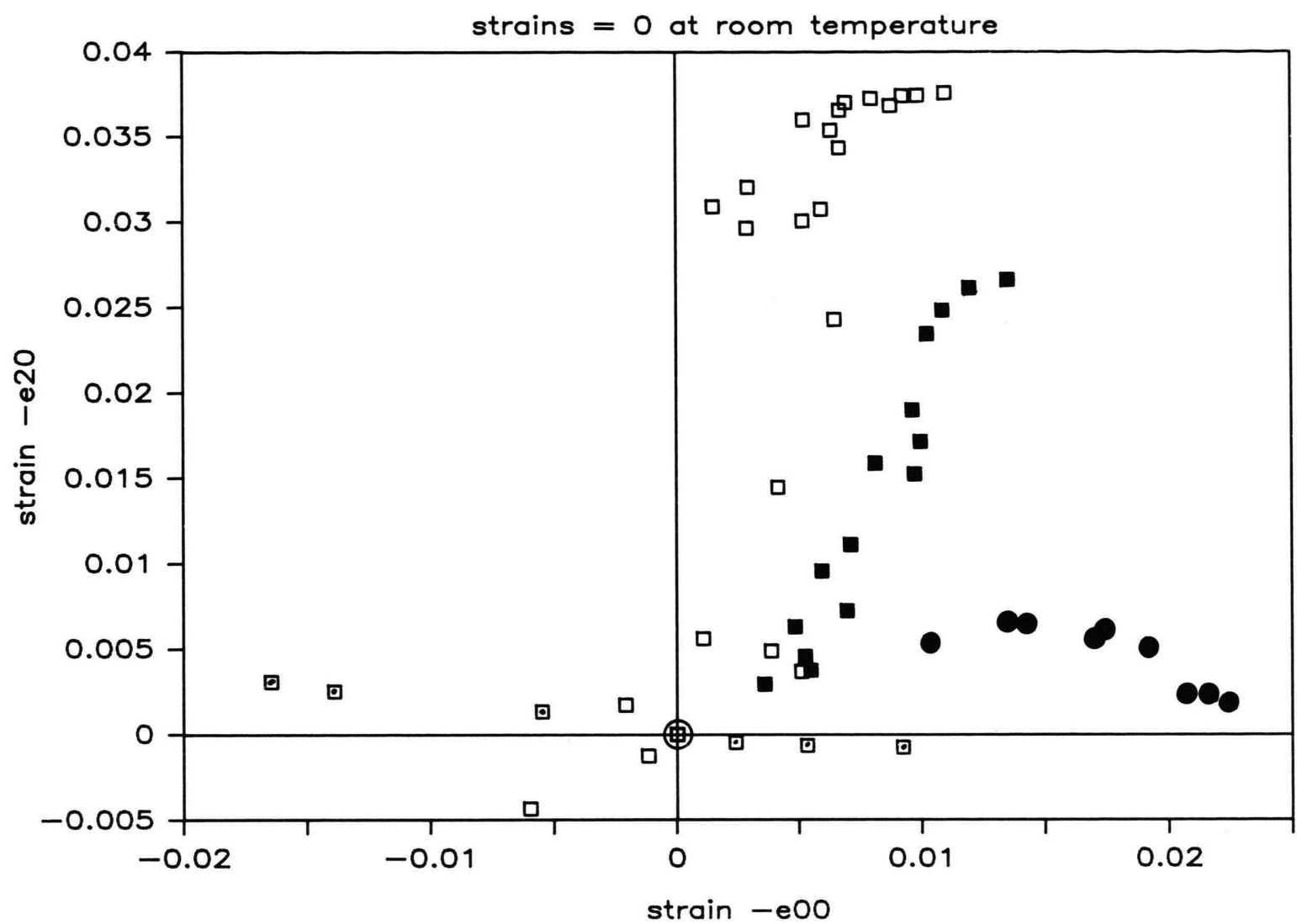

Fig. 2. The thermal expansion behaviour of some scheelite-structure crystals. The distortion, measured by $-\varepsilon_{20}$, is plotted vertically against the negative volume change, $-\varepsilon_{00}$. For consistency between compounds, the strains have been calculated relative to the unit cell dimensions at $25^{\circ} \mathrm{C}$, which results in slightly different numbers from those in [49]; the point at the origin is common to all four sets of data. In general, temperature is increasing from right to left. Legend: open squares, $\mathrm{NH}_{4} \mathrm{ReO}_{4}$; filled squares, $\mathrm{NH}_{4} \mathrm{IO}_{4}$; filled circles, $\mathrm{NH}_{4} \mathrm{TcO}_{4}$; squares with points, $\mathrm{KReO}_{4}$.

where $2 \Delta_{0}$ is the difference in energy between the two orientations and $c_{0}$ is the elastic constant corresponding to the strain $\varepsilon_{20}$. The last terms represent the elastic strain energy. The summation is over unit cells, each of which contains two ammonium ions. Minimization of free energy with respect to strain gives the result $\varepsilon_{20}=2 V_{s}\left\langle\sigma^{z}\right\rangle / \Omega c_{0}$; if this is substituted back into (17), and direct interactions are included as a parameter $J(n-m)$, there results

$$
\begin{aligned}
\mathscr{H}= & -\sum_{n} \Delta_{0} \sigma^{z}(n) \\
& -\frac{1}{2} \sum_{n m}\left\{J(n-m)+V_{s}^{2} / N \Omega c_{0}\right\} \sigma^{z}(n) \sigma^{z}(m) .
\end{aligned}
$$

This is now in a form to which the mean field approximation can be applied, and the result yields explicit forms for the strain, the heat capacity and elastic con- stant as functions of temperature. There are two adjustable parameters which can be determined from the temperature dependence of the strain, namely $\Delta_{0}$ (the energy difference between the two orientations at zero strain), and the effective coupling between ions,

$$
J^{\prime}=V_{s}^{2} / \Omega c_{0}+\sum J(n-m) .
$$

These parameters are found to be (in temperature units) $\Delta_{0}=9.0 \mathrm{~K}$ and $J^{\prime}=210 \mathrm{~K}$, respectively. The NQR temperature dependence is well described by these parameters, and $v$ is found to be a linear function of the strain $\varepsilon_{20}$. A peak in the heat capacity at $200 \mathrm{~K}$ with a magnitude of about $R$ is predicted; the theory is a constant stress theory and therefore can be compared directly with the measured $C_{p}$ rather than $C_{v}$ or $C_{\varepsilon}$ [26]. The experimental value of the ammonium ion heat capacity at constant stress is about $7 R$ [26], but 
this includes the rotational kinetic energy; further examination of this discrepancy is required. A softening of the elastic constant near $200 \mathrm{~K}$ is predicted, but no data are presently available for comparison.

The theory is subject to further experimental test through measurements of the elastic constants over the whole temperature range, and by direct examination of the basic postulate that the equatorial orientation of the ammonium ion becomes populated at high temperature. The earlier neutron diffraction data [51] were not clear on the latter point, although the question was examined during data refinement. These studies are in progress. No consideration has been given to the pressure dependence of $v[55,56]$, or to the temperature dependent deuteration shift [57], and the apparent relationship between these two.

The extension of the theory to salts other than $\mathrm{NH}_{4} \mathrm{ReO}_{4}$ is essential if it is to be of general interest, for the three known ammonium scheelites examined so far are strikingly similar. Figure 2 shows a graph of $\varepsilon_{20}$ versus $\varepsilon_{00}$ for the three ammonium salts, plus $\mathrm{KReO}_{4}$, for a range of temperatures. It can be seen that in the periodate and pertechnetate, $\varepsilon_{00}$ varies considerably as well as $\varepsilon_{20}$, and so extension of the

[1] H. Bayer, Z. Physik 130, 227 (1951).

[2] T. Kushida, J. Sci. Hiroshima Univ. A 19, 327 (1955).

[3] T. C. Wang, Phys. Rev. 99, 566 (1955).

[4] T. Kushida, G. B. Benedek, and N. Bloembergen, Phys. Rev. 104, 1364 (1956).

[5] R. J. C. Brown, J. Chem. Phys. 32, 116 (1960).

[6] D. Nakamura, R. Ikeda, and M. Kubo, Coord. Chem. Rev. 17, 281 (1975).

[7] H. Chihara and N. Nakamura, Adv. in NQR, Vol. 4, p. 1, Heyden, London 1980.

[8] S. Segel, private communication.

[9] G. C. Gillies and R. J. C. Brown, Adv. in NQR, Vol. 1, p. 329, Heyden, London 1974.

[10] R. J. C. Brown, J. Mol. Struct. 58, 85 (1980).

[11] K. Negita, N. Nakamura, and H. Chihara, Chem. Phys. Lett. 63, 187 (1979).

[12] H. Chihara, K. Negita, Y. Yoshioka, and N. Nakamura, J. Mol. Struct. 58, 155 (1980).

[13] C. Dimitropoulos and J. Pelzl, J. Mol. Struct. 58, 107 (1980).

[14] R. J. C. Brown, B. K. Hunter, M. Mackowiak, and S. Segel, J. Magn. Reson. 50, 218 (1982).

[15] Y. Yoshioka, N. Nakamura, and H. Chihara, J. Chem. Soc. Faraday Trans. 2, 79, 497 (1983).

[16] M. Mackowiak and R. J. C. Brown, J. Mol. Struct. 111, 233 (1983).

[17] N. Nakamura, Z. Naturforsch. 41 a, 243 (1986).

[18] Y. Furukawa and D. Nakamura, Ber. Bunsenges. Phys. Chem. 93, 13 (1989).

[19] M. Mizuno, T. Asaji, and D. Nakamura, Z. Naturforsch. 44 a, 210 (1989).

[20] R. M. Richardson and J. Howard, Chem. Phys. 86, 235 (1984). theory may require inclusion of the second strain variable. Further, it can be seen that in the pertechnetate, $\varepsilon_{20}$ passes through a maximum at about $200 \mathrm{~K}$ while the quadrupole frequency is a smooth function of temperature with a broad maximum centred at about $300 \mathrm{~K}[8,58]$. The heat capacity of the periodate has been measured and can be used for comparison with the theory [59]. The orthorhombic salts may not fit the framework of the theory at all because the ammonium ion site is of low symmetry and there are no two obvious orientations.

Thus, while the pseudo-spin theory is apparently very successful in fitting the one case for which it was developed, its general applicability to ammonium salts has yet to be established.

\section{Acknowledgements}

This work was supported financially by the Natural Sciences and Engineering Research Council of Canada. The support and cooperation of many colleagues has been essential to this work and is gratefully acknowledged.

[21] R. Ikeda and C. A. McDowell, Chem. Phys. Lett. 14, 389 (1972).

[22] D. A. Johnson, J. Chem. Soc. Chem. Comm. 1986, 534.

[23] D. A. Johnson, J. Chem. Soc. Dalton Trans. 1988, 445.

[24] Y. S. Park, H. F. Shurvell, and R. J. C. Brown, J. Raman Spectrosc. 17, 351 (1986).

[25] R. L. Armstrong, J. A. J. Lourens, and K. R. Jeffrey, J. Magn. Reson. 23, 115 (1976).

[26] R. J. C. Brown, J. E. Callanan, R. D. Weir, and E. F. Westrum, Jr., J. Chem. Phys. 85, 5963 (1986).

[27] S. L. Segel, R. J. C. Brown, and R. D. Heyding, J. Chem. Phys. 69, 3435 (1978).

[28] R. J. C. Brown, J. Magn. Reson. 42, 1 (1981).

[29] R. Faggiani, C. J. L. Lock, and J. Poce, Acta Cryst. B 36, 231 (1980).

[30] P. K. Burkert, J. Mol. Struct. 83, 307 (1982).

[31] P. K. Burkert and D. G. Klobasa, Z. Naturforsch. 40 a, 274 (1985).

[32] D. G. Klobasa and P. K. Burkert, Mag. Res. in Chem. 25, 154 (1987).

[33] D. G. Klobasa and P. K. Burkert, Z. Naturforsch. 42 a, 275 (1987).

[34] P. K. Burkert and M. Grommelt, Z. Naturforsch. 43 b, 1381 (1988).

[35] T. Kitazume, M. Sekizaki, and M. Suhara, J. Mol. Struct. 58, 161 (1980).

[36] K. Horiuchi, A. Sasane, Y. Moti, T. Asaji, and D. Nakamura, Bull. Chem. Soc. Japan 59, 2639 (1986).

[37] T. J. Bastow, private communication.

[38] R. J. C. Brown and S. L. Segel, J. Magn. Reson. 45, 284 (1981).

[39] Y. M. Seo, J. Pelzl, and C. Dimitropoulos, Z. Naturforsch. 41 a, 311 (1986). 
[40] C. Dimitropoulos and J. Pelzl, Z. Naturforsch. 44a, 109 (1989).

[41] B. M. Powell, W. Press, and G. Dolling, Phys. Rev. B 32, 3118 (1985).

[42] H. M. Van Driel and R. L. Armstrong, Can. J. Phys. 53, 1141 (1975).

[43] P. Storck and Al. Weiss, Ber. Bunsenges. Phys. Chem. 93, 454 (1989)

[44] A. Ishikawa, Y. Ito, K. Horiuchi, T. Asaji, and D. Nakamura, J. Mol. Struct. 192, 221 (1989).

[45] Y. Ito, T. Asaji, R. Ikeda, and D. Nakamura, Ber. Bunsenges. Phys. Chem. 92, 885 (1988).

[46] D. Borchers and Al. Weiss, Z. Naturforsch. 42 a, 739 (1987).

[47] K. B. Dillon, J. Halfpenny, and A. Marshall, J. Chem. Soc. Dalton Trans. 1985, 1399.

[48] Y. Tai, T. Asaji, R. Ikeda, and D. Nakamura, Z. Naturforsch. 44 a, 300 (1989).

[49] D. R. Taylor, J. Chem. Phys. 87, 773 (1987).
[50] D. R. Taylor, Phys. Rev. B 40, 493 (1989).

[51] R. J. C. Brown, S. L. Segel, and G. Dolling, Acta Cryst. B 36, 2195 (1980).

[52] D. Smith, J. Chem. Phys. 79, 2995 (1983).

[53] R. J. C. Brown, J. G. Smeltzer, and R. D. Heyding, J. Magn. Reson. 24, 269 (1976).

[54] S. N. Stuart, CSIRO Melbourne, private communication.

[55] R. J. C. Brown and S. L. Segel, J. Chem. Phys. 67, 3163 (1977).

[56] S. L. Segel and R. J. C. Brown, J. Chem. Phys. 70, 3840 (1979).

[57] T. L. Haslett, R. J. C. Brown, Y. S. Park, and H. F. Shurvell, Z. Naturforsch. 41 a, 335 (1986).

[58] V. P. Tarasov, S. A. Petrushin, V. I. Privalov, K. E. German, S. V. Kryuchov, and Yu. A. Buslaev, Soviet. J. Coord. Chem. 12, 713 (1986).

[59] R. J. C. Brown, J. E. Callanan, R. D. Weir, and E. F. Westrum, Jr., J. Chem. Thermod. 19, 1173 (1987). 